\title{
Automorphism groups of some variants of lattices
}

\begin{abstract}
Ganyushkin O.G., Desiateryk O.O. ${ }^{\bowtie}$
In this paper we consider variants of the power set and the lattice of subspaces and study automorphism groups of these variants. We obtain irreducible generating sets for variants of subsets of a finite set lattice and subspaces of a finite vector space lattice.

We prove that automorphism group of the variant of subsets of a finite set lattice is a wreath product of two symmetric permutation groups such as first of this groups acts on subsets. The automorphism group of the variant of the subspace of a finite vector space lattice is a natural generalization of the wreath product. The first multiplier of this generalized wreath product is the automorphism group of subspaces lattice and the second is defined by the certain set of symmetric groups.

Key words and phrases: lattice, variant, sandwich semigroup, automorphism group, power set, subspaces lattice, generating set.
\end{abstract}

Taras Shevchenko National University of Kyiv, 64/13 Volodymyrska str., 01601, Kyiv, Ukraine

$\checkmark$ Corresponding author

E-mail: ganiyshk@gmail.com (Ganyushkin O.G.), sasha.desyaterik@gmail.com (Desiateryk O.O.)

\section{Introduction}

Let $S$ be a semigroup and $a \in S$. A binary operation $*_{a}$ defined on the set $S$ by $x *_{a} y=x a y$, $x, y \in S$, is associative. This operation $*_{a}$ is called a sandwich operation and the semigroup $\left(S, *_{a}\right)$ is called a variant of $S$ or a sandwich semigroup.

E.S. Lyapin initiated the study of variants in his monograph [1]. In that work he studied transformation semigroups. Variants of other types of semigroups were studied by various authors, for example, papers [2-5], chapter 13 in monograph [6] and references in this chapter. We studied variants of commutative bands with zero in [7] and variants of a lattice of partitions of a countable set in [8].

We study variants of lattices which are considered as semigroups with respect to the operation $\wedge$ of taking the greatest lower bound of two elements. Note, that the inequality $a \leqslant b$ holds if and only if $a \wedge b=a$. Besides, the transformation $\varphi: L \rightarrow L$ is an automorphism of the lattice $L$ as an ordered set $(L, \leqslant)$ if and only if it is an automorphism of $L$ as a semigroup $(L, \wedge)$.

Let $(L, \leqslant)$ be a lower semilattice. For any $a \in L$ by $\Delta_{a}=\{x \in L: x \wedge a=x\}$ we define the lower conus of the element $a$.

Proposition 1. Each generating set of the variant $\left(L, *_{a}\right)$ contains the set $L \backslash \Delta_{a}$.

Proof. Let an element $x$ be decomposable in the variant $\left(L, *_{a}\right)$. Then there exist elements 
$y, z \in L$ such that $x=y *_{a} z=y \wedge a \wedge z$. However,

$$
x \wedge a=y \wedge a \wedge z \wedge a=y \wedge a \wedge z=x
$$

in the other words $x \in \Delta_{a}$. Hence all elements from $L \backslash \Delta_{a}$ are not decomposable, so they belong to each generating system.

Theorem 1. For each automorphism $\varphi \in \operatorname{Aut}\left(L, *_{a}\right)$ the lower conus $\Delta_{a}$ is an invariant subset. Also, the restriction $\varphi$ to $\Delta_{a}$ is an automorphism of $\Delta_{a}$ as an partially ordered set.

Proof. Note, that $\varphi(a) \leq a$. Obviously,

$$
\varphi(a)=\varphi(a \wedge a \wedge a)=\varphi\left(a *_{a} a\right)=\varphi(a) *_{a} \varphi(a)=\varphi(a) \wedge a \wedge \varphi(a)=a \wedge \varphi(a) .
$$

Hence, $\varphi(a) \leqslant a$.

Further, we have to show, that lower conus $\Delta_{a}$ is an invariant set for $\varphi$. Evidently, if $x \leq a$, then $x=x \wedge a \wedge a=x *_{a} a$. Hence,

$$
\varphi(x)=\varphi\left(x *_{a} a\right)=\varphi(x) *_{a} \varphi(a)=\varphi(x) \wedge a \wedge \varphi(a)=\varphi(x) \wedge \varphi(a) \leq \varphi(a) \leq a .
$$

Suppose $x \leq y \leq a$. Then $x=x \wedge a \wedge y=x *_{a} y$. It follows

$$
\varphi(x)=\varphi(x) *_{a} \varphi(y)=\varphi(x) \wedge a \wedge \varphi(y)=\varphi(x) \wedge \varphi(y)
$$

Hence, $\varphi(x) \leq \varphi(y)$ and $\varphi$ preserves the partial order on $\Delta_{a}$.

Thus, the restriction $\left.\varphi\right|_{\Delta_{a}}: \Delta_{a} \rightarrow \Delta_{a}$ is a homomorphism of partially ordered sets. Since inverse mapping $\varphi^{-1}$ also belongs to $\operatorname{Aut}\left(L, *_{a}\right)$, it follows that $\left.\varphi\right|_{\Delta_{a}}$ is a bijection and hence it is an automorphism of $\Delta_{a}$ as a partially ordered set.

Corollary 1. For any automorphism $\varphi \in \operatorname{Aut}\left(L, *_{a}\right)$ we have $\varphi(a)=a$.

Lemma 1. An element $b \in L$ is an idempotent of the semigroup $\left(L, *_{a}\right)$ if and only if $b \in \Delta_{a}$.

Proof. Let $b$ be an idempotent of the semigroup $\left(L, *_{a}\right)$. Then

$$
b=b *_{a} b=b \wedge a \wedge b=b \wedge a, \text { that is } b \leq a .
$$

Conversely, if $b \leq a$, then $b=b \wedge a=b \wedge a \wedge b=b *_{a} b$. Hence, $b$ is an idempotent of the semigroup $\left(L, *_{a}\right)$.

Lemma 2. For any $b \in L$ and $\varphi \in \operatorname{Aut}\left(L, *_{a}\right)$, we have $\varphi(b \wedge a)=\varphi(b) \wedge a$.

Proof. The statement of the lemma follows from the chain of equalities

$$
\varphi(b \wedge a)=\varphi(b \wedge a \wedge a)=\varphi\left(b *_{a} a\right)=\varphi(b) *_{a} \varphi(a)=\varphi(b) \wedge a \wedge \varphi(a)=\varphi(b) \wedge a,
$$

the last equality is true by the Corollary 1. 


\section{Power set}

Let $M$ be a finite set. We define a power set of $M$ as an ordered by inclusion set $\mathfrak{B}(M)$ of all subsets of the set $M$.

Proposition 2. For any proper subset $A \subset M$ the set $\mathfrak{B}(M) \backslash \mathfrak{B}(A)$ is an irreducible system of generators of the variant $\left(\mathfrak{B}(M), *_{A}\right)$.

Proof. The lower conus $\triangle_{A}$ of the power set $\mathfrak{B}(M)$ coincides with $\mathfrak{B}(A)$. Thus, by the Proposition 1 , each generating set of the variant $\left(\mathfrak{B}(M), *_{A}\right)$ contains $\mathfrak{B}(M) \backslash \mathfrak{B}(A)$. Further, we only have to show that $\mathfrak{B}(M) \backslash \mathfrak{B}(A)$ is a generating set of the variant $\left(\mathfrak{B}(M), *_{A}\right)$. For two arbitrary $B \in \mathfrak{B}(A)$ and $b \notin A$ we have $B \cup\{b\} \notin \mathfrak{B}(A)$ and

$$
(B \cup\{b\}) *_{A}(B \cup\{b\})=(B \cup\{b\}) \cap A \cap(B \cup\{b\})=B .
$$

It implies that each element from $\mathfrak{B}(A)$ is also decomposable to a product of elements from $\mathfrak{B}(M) \backslash \mathfrak{B}(A)$.

For each transformation $g: a \mapsto a^{g}$ of the finite set $M$ there exists the induced transformation $\widehat{g}:\left\{a_{1}, \ldots, a_{k}\right\} \mapsto\left\{a_{1}^{g}, \ldots, a_{k}^{g}\right\}$ of the power set $\mathfrak{B}(M)$.

Proposition 3. Let $\widehat{S_{n}}$ be an induced action of the symmetric group $S_{n}$ on the subsets of $N=\{1,2, \ldots, n\}$. Then group $\widehat{S_{n}}$ is an automorphism group of the power set of the set $N$.

Proof. Let subsets $A, B \subseteq\{1,2, \ldots, n\}$ be such as

$$
A \cap B=\left\{c_{1}, \ldots, c_{k}\right\}, A=\left\{a_{1}, \ldots, a_{m}, c_{1}, \ldots, c_{k}\right\}, B=\left\{b_{1}, \ldots, b_{r}, c_{1}, \ldots, c_{k}\right\} .
$$

Then for every permutation $\pi \in S_{n}$ we have

$$
\pi(A)=\left\{\pi\left(a_{1}\right), \ldots, \pi\left(a_{m}\right), \pi\left(c_{1}\right), \ldots, \pi\left(c_{k}\right)\right\}, \pi(B)=\left\{\pi\left(b_{1}\right), \ldots, \pi\left(b_{r}\right), \pi\left(c_{1}\right), \ldots, \pi\left(c_{k}\right)\right\},
$$

and since $\pi$ is injective, we obtain

$$
\pi(A) \cap \pi(B)=\left\{\pi\left(c_{1}\right), \ldots, \pi\left(c_{k}\right)\right\}=\pi(A \cap B) .
$$

Hence, the induced action $\pi$ on $\mathfrak{B}(N)$ is an automorphism and $\widehat{S_{n}} \subseteq \operatorname{Aut}(\mathfrak{B}(N))$.

Conversely, we consider $\varphi \in \operatorname{Aut}(\mathfrak{B}(N))$. Since $\varphi$ maps atoms into atoms, automorphism $\varphi$ induces permutation $\pi_{\varphi}=\left(\begin{array}{cccc}1 & 2 & \cdots & n \\ \varphi(1) & \varphi(2) & \cdots & \varphi(n)\end{array}\right) \in S_{n}$. Each element $A=\left\{a_{1}, \ldots, a_{m}\right\}$ of the boolean $\mathfrak{B}(N)$ is fully defined by the set of smaller atoms $\left\{a_{1}\right\}, \ldots,\left\{a_{m}\right\}$. Thus, $\varphi(A)=\left\{\varphi\left(a_{1}\right), \cdots, \varphi\left(a_{m}\right)\right\}$, then $\varphi$ coincides with the induced by the permutation $\pi_{\varphi}$ automorphism. Hence, $\widehat{S_{n}} \supseteq \operatorname{Aut}\left(\mathfrak{B}_{n}\right)$.

Theorem 2. If $|M|=n, A \subseteq M$ and $|A|=k$, then automorphism group $\operatorname{Aut}\left(\mathfrak{B}(M), *_{A}\right)$ of the variant $\left(\mathfrak{B}(M), *_{A}\right)$ is isomorphic to the wreath product $\widehat{S_{k}}<S_{2^{n-k_{-}}}$of two permutation groups $\widehat{S}_{k}$ and $S_{2^{n-k}-1}$.

Proof. If $A=M$, then $n=k$ and the wreath product $\widehat{S_{k}} 2 S_{2^{n-k}-1}$ coincides with the group $\widehat{S_{n}}$. Thus, further we consider $A$ as a proper subset of $M$.

The element $B \subseteq M$ of the semigroup $\left(\mathfrak{B}(M), *_{A}\right)$ is an idempotent if and only if $B \subseteq A$. Moreover, idempotents generate subsemigroup $\left(\mathfrak{B}(A), *_{A}\right)$ and it coincides with $(\mathfrak{B}(A), \cap)$. 
Hence, the automorphism $\varphi \in \operatorname{Aut}\left(\mathfrak{B}(M), *_{A}\right)$ maps the subsemigroup $\left(\mathfrak{B}(A), *_{A}\right)$ to itself. Furthermore, the restriction $\widetilde{\varphi}$ of the automorphism $\varphi$ to the semigroup $\left(\mathfrak{B}(A), *_{A}\right)$ is an automorphism $\left(\mathfrak{B}(A), *_{A}\right)$. Besides, $A$ and $\varnothing$ are fixed points (as 1 and 0 of this semigroup). It follows that $\varphi(A)=A$ and $\varphi(\varnothing)=\varnothing$.

Further, we represent each element $B \in \mathfrak{B}(M) \backslash \mathfrak{B}(A)$ as a disjoint union $B=B_{1} \dot{\cup} B_{2}$, where $B_{1}=B \cap A$ and $B_{2}=B \backslash A$. Moreover, $B_{1}=A *_{A} B$ and $B_{2} \neq \varnothing$. It follows that

$$
\varphi\left(B_{1}\right)=\varphi(A) *_{A} \varphi(B)=A \cap \varphi(B) .
$$

Thus, $\varphi(B)=\varphi\left(B_{1}\right) \dot{\cup} B^{\prime}$, where $B^{\prime} \neq \varnothing$. Hence, automorphism $\varphi$ maps all elements of $B \in \mathfrak{B}(M) \backslash \mathfrak{B}(A)$ with a fixed first component $B_{1}$ to the elements with the fixed first component $\varphi\left(B_{1}\right)$. Furthermore, for each fixed subset $B_{1} \subseteq A$ the automorphism $\varphi$ induces a permutation $\pi_{B_{1}}: B_{2} \mapsto B^{\prime}$ of non empty sets of the set $M \backslash A$. Moreover, since $\varphi \in \operatorname{Aut}\left(\mathfrak{B}(M), *_{A}\right)$, it follows that

$$
\varphi\left(\left(B_{1} \dot{\cup} B_{2}\right) *_{A}\left(C_{1} \dot{\cup} C_{2}\right)\right)=\varphi\left(\left(B_{1} \dot{\cup} B_{2}\right)\right) *_{A} \varphi\left(\left(C_{1} \dot{\cup} C_{2}\right)\right) .
$$

Let $G=(\operatorname{Aut}(\mathfrak{B}(A)), \mathfrak{B}(A))$ be a permutation group. Thus, $G$ is similar to $\widehat{S_{k}}$ as a permutation group. Let $H$ be a symmetric group of all permutations on the set $\mathfrak{B}(M \backslash A) \backslash\{\varnothing\}$ of all non empty subsets of the set $M \backslash A$. Obviously, $H$ is similar to $S_{2^{n-k}-1}$ as a group of permutations. Let $\widetilde{\varphi} \in G$ be a restriction of $\varphi$ to $\mathfrak{B}(A)$. Let $f_{\varphi}$ be a function from $\mathfrak{B}(A)$ to $H$ such that $f_{\varphi}$ set a correspondence of each subset $B_{1} \subseteq A$ to a permutation $f_{\varphi}\left(B_{1}\right)=\pi_{B_{1}}$. From the above it follows that each automorphism $\varphi \in \operatorname{Aut}\left(\mathfrak{B}(M), *_{A}\right)$ induces a pair $\left(\widetilde{\varphi}, f_{\varphi}\right)$. Since $\mathfrak{B}(M)=\mathfrak{B}(A) \times \mathfrak{B}(M \backslash A)$, it follows that the pair $\left(\widetilde{\varphi}, f_{\varphi}\right)$ is an element of the wreath product $G$ ? $H$ of two groups $G$ and $H$.

Therefore, we find mapping $\varphi \mapsto\left(\widetilde{\varphi}, f_{\varphi}\right)$ of the permutation group $\left(\operatorname{Aut}\left(\mathfrak{B}(M), *_{A}\right), \mathfrak{B}(M)\right)$ into the wreath product $G ? H$. Further, we have to show that this mapping is surjective.

Consider any permutation $\varphi \in G \imath H$, that is a pair of an automorphism $\mu \in \operatorname{Aut}(\mathfrak{B}(A))$ and a function $f: \mathfrak{B}(A) \rightarrow H$. The permutation $\varphi$ acts on elements from $\mathfrak{B}(M)$ by the next rule

$$
\varphi(B)= \begin{cases}\mu(B), & \text { if } B \subseteq A ; \\ \mu\left(B_{1}\right) \dot{\cup} f\left(B_{1}\right)\left(B_{2}\right), & \text { if } B \nsubseteq A \text { and } B=B_{1} \dot{\cup} B_{2},\end{cases}
$$

here $B_{1}=B \cap A$ and $B_{2}=B \backslash A$. Hence, for each $B, C \in \mathfrak{B}(M)$

$$
f\left(B_{1}\right)\left(B_{2}\right) \cap A=f\left(C_{1}\right)\left(C_{2}\right) \cap A=\varnothing,
$$

it follows that

$$
\begin{aligned}
& \varphi\left(B *_{A} C\right)=\varphi(B \cap A \cap C)=\varphi\left(B_{1} \cap C_{1}\right)=\mu\left(B_{1} \cap C_{1}\right)=\mu\left(B_{1}\right) \cap \mu\left(C_{1}\right), \\
& \qquad(B) *_{A} \varphi(C)=\left(\mu\left(B_{1}\right) \dot{\cup} f\left(B_{1}\right)\left(B_{2}\right)\right) *_{A}\left(\mu\left(C_{1}\right) \dot{\cup} f\left(C_{1}\right)\left(C_{2}\right)\right) \\
& =\mu\left(B_{1}\right) \cap A \cap \mu\left(C_{1}\right)=\mu\left(B_{1}\right) \cap \mu\left(C_{1}\right) .
\end{aligned}
$$

Therefore, $\varphi$ is the automorphism of the variant $\left(\mathfrak{B}(M), *_{A}\right)$.

\section{The lattice of subspaces}

Let $V$ be a $n$-dimensional vector space over the field $\mathbb{F}_{q}$ of the order $q$. Let $\mathcal{L}(V)$ be an ordered by inclusion lattice of the subspaces of the space $V$. Let $\left(\mathcal{L}(V), *_{A}\right)$ be a variant of the semigroup $(\mathcal{L}(V), \cap)$ generated by the fixed subspace $A \subseteq V$ such as $\operatorname{dim} A=m$. 
Proposition 4. For any proper subspace $A \subset V$ the set $\mathcal{L}(V) \backslash \mathcal{L}(A)$ is an irreducible system of generators of the variant $\left(\mathcal{L}(V), *_{A}\right)$.

Proof. The proof is similar to the proof of the Proposition 2.

Lemma 3. Let $B$ be a fixed subspace of $A$ and $\operatorname{dim} B=k \leq m$. Then a number of $t$-dimensional subspaces $U \in \mathcal{L}(V)$ of $U \cap A=B$ is equal to

$$
\frac{\left(q^{n}-q^{m}\right)\left(q^{n}-q^{m+1}\right) \cdots\left(q^{n}-q^{m+t-k-1}\right)}{\left(q^{t}-q^{k}\right)\left(q^{m}-q^{k+1}\right) \cdots\left(q^{t}-q^{t-1}\right)}
$$

Proof. Let $\boldsymbol{e}_{1}, \ldots, \boldsymbol{e}_{k}$ be some base of $B$. We complement it to the base $\boldsymbol{e}_{1}, \ldots, \boldsymbol{e}_{k}, \boldsymbol{e}_{k+1}, \ldots, \boldsymbol{e}_{t}$ of some subspace from the set

$$
\mathcal{M}=\{U \subseteq V \mid \operatorname{dim} U=t, U \cap A=B\} .
$$

Since vectors $\boldsymbol{e}_{k+1}, \ldots, \boldsymbol{e}_{t}$ lay in the subspace $V \backslash A$, the above base can be complemented in $\left(q^{n}-q^{m}\right)\left(q^{n}-q^{m+1}\right) \ldots\left(q^{n}-q^{m+t-k-1}\right)$ ways. Necessity of this fact is obvious. Further, if

$$
\alpha_{1} \boldsymbol{e}_{1}+\cdots+\alpha_{k} \boldsymbol{e}_{k}+\alpha_{k+1} \boldsymbol{e}_{k+1}+\cdots+\alpha_{t} \boldsymbol{e}_{t} \in A,
$$

then $\alpha_{k+1} \boldsymbol{e}_{k+1}+\cdots+\alpha_{t} \boldsymbol{e}_{t} \in A$ and from the choice of vectors it follows $\alpha_{k+1}=\cdots=\alpha_{t}=0$. Hence, sufficiency is proved. Furthermore, a number of possibilities to compliment $e_{1}, \ldots, \boldsymbol{e}_{k}$ to the base of some defined space from $\mathcal{M}$ is equals to $\left(q^{t}-q^{k}\right)\left(q^{m}-q^{k+1}\right) \cdots\left(q^{t}-q^{t-1}\right)$.

Denote the number (1) by $a_{t, k}^{n, m}$. To finalize the main result about the structure of the automorphism group of the variant $\left(\mathcal{L}(V), *_{A}\right)$ we need the next construction.

Let $(G, M)$ be permutation group such that $O_{1}, \ldots, O_{k}$ are orbits of this group. Let $\left(H_{1}, N_{1}\right), \ldots,\left(H_{k}, N_{k}\right)$ be permutation groups.

Let $g$ be an element from the group $G$. For each $i, 1 \leq i \leq k$, let $f_{i}$ be a function from $O_{i}$ to $H_{i}$. Then by $G ?\left(H_{1}, \ldots, H_{k}\right)$ we denote the generalized wreath product, that is a permutation group such that sets $\left(g, f_{1}, \ldots, f_{k}\right)$ are elements of this group. The group $G ?\left(H_{1}, \ldots, H_{k}\right)$ acts on the set $\bigcup_{i=1}^{k}\left(O_{i} \times N_{i}\right)$ by the next rule

$$
(m, n)^{\left(g, f_{1}, \ldots, f_{k}\right)}=\left(m^{g}, n^{f_{i}(g)}\right) \quad \text { if } m \in N_{i} .
$$

Theorem 3. Let $A$ be an $m$-dimensional subspace of an $n$-dimensional vector space $V$ over the field $\mathbb{F}_{q}$ of the order $q$. The automorphism group of the variant $\left(\mathcal{L}(V), *_{A}\right)$ is isomorphic to the generalized wreath product

$$
S=\operatorname{Aut}(\mathcal{L}(A)) \imath\left(S_{r_{0}}, S_{r_{1}}, \ldots, S_{r_{m}}\right),
$$

where $r_{k}=\sum_{t=k+1}^{n} a_{t, k}^{n, m}$.

Proof. If $A=V$, then $m=n, r_{0}=r_{1}=\ldots=r_{m}=1$ and generalized wreath product $S$ coincides with the group $\operatorname{Aut}(\mathcal{L}(A))$. Thus, further we can consider $A$ as a proper subspace of the space $V$.

The element $U \subseteq V$ of the semigroup $\left(\mathcal{L}(V), *_{A}\right)$ is an idempotent if and only if $U \subseteq A$. Moreover, idempotents generate a subsemigroup of $\left(\mathcal{L}(A), *_{A}\right)$ and this subsemigroup coincides with $(\mathcal{L}(A), \cap)$. It follows that the automorphism $\varphi \in \operatorname{Aut}\left(\mathcal{L}(V), *_{A}\right)$ maps the subsemigroup $\left(\mathcal{L}(A), *_{A}\right)=(\mathcal{L}(A), \cap)$ to itself. 
In addition, the restriction $\widetilde{\varphi}$ of the automorphism $\varphi$ on subsemigroup $(\mathcal{L}(A), \cap)$ is an automorphism of the semigroup $(\mathcal{L}(A), \cap)$ such that $A$ and $\{0\}$ are fixed points (as 1 and 0 of this subsemigroup).

Consider the set

$$
\widetilde{U}=\{W \backslash V: W \nsubseteq A \text { and } W \cap A=U\}
$$

for each subspace $U \subseteq A$.

Note that $|\widetilde{U}|=r_{\operatorname{dim} U}$. We enumerate elements of each set $\widetilde{U}$, that is,

$$
\widetilde{U}=\left\{X_{1}^{(U)}, X_{2}^{(U)}, \ldots, X_{r_{\operatorname{dim} U}}^{(U)}\right\} .
$$

We represent each element $U \in \mathcal{L}(V) \backslash \mathcal{L}(A)$ in the form $U=U_{1} \cup U_{2}$ such that $U_{1}=U \cap A$ and $U_{2}=U \backslash A$. Moreover, $U_{1}=A *_{A} U$ and $U_{2} \neq \varnothing$. Hence,

$$
\varphi\left(U_{1}\right)=\varphi(A) *_{A} \varphi(U)=A \cap \varphi(U) .
$$

It follows that $\varphi(U)=\varphi\left(U_{1}\right) \dot{\cup} U^{\prime}$, such as $U^{\prime} \neq \varnothing$. Thus, automorphism $\varphi$ maps all elements $U \in \mathcal{L}(V) \backslash \mathcal{L}(A)$ with the first fixed component $U_{1}$ to elements with the fixed first component $\varphi\left(U_{1}\right)$.

Since the restriction $\varphi$ to $\mathcal{L}(A)$ is an automorphism of the lattice $\mathcal{L}(A)$, then $\varphi$ preserves the ranks of elements from $\mathcal{L}(A)$. Thus, $\operatorname{dim} U_{1}=\operatorname{dim} \varphi\left(U_{1}\right)$. Furthermore, for elements with the fixed first component $U_{1}$, the automorphism $\varphi$ induces a bijection

$$
f_{\varphi}^{\left(U_{1}\right)}: \widetilde{U_{1}} \mapsto \widetilde{\varphi\left(U_{1}\right)}, \quad U_{2} \mapsto U^{\prime} .
$$

With respect to the numeration of sets $\widetilde{U_{1}}$ and $\widetilde{\varphi\left(U_{1}\right)}$, the bijection $f_{\varphi}^{\left(U_{1}\right)}$ define some permutation $\pi^{\left(U_{1}\right)}(\varphi) \in S_{r_{\operatorname{dim} U_{1}}}$.

The automorphism group $\operatorname{Aut}(\mathcal{L}(A)$ preserves subspaces dimensions. On the other hand, $\operatorname{Aut}(\mathcal{L}(A))$ contains the projective group $P G L(A)$, such that $\operatorname{PGL}(A)$ acts transitive on each set $O_{k}=\{W \subseteq A: \operatorname{dim} W=k\}$. Thus, $O_{0}, O_{1}, \ldots, O_{m}$ are group orbits.

Let the function $f_{\varphi}^{(k)}: O_{k} \rightarrow S_{r_{k}}$ be defined for each $k=0,1, \ldots, m$ as $f_{\varphi}^{(k)}(W)=\pi^{(W)}(\varphi)$. Then for each automorphism $\varphi \in \operatorname{Aut}\left(\mathcal{L}(V), *_{A}\right)$ we define a set

$$
T_{\varphi}=\left(\widetilde{\varphi}, f_{\varphi}^{(0)}, f_{\varphi}^{(1)}, \ldots, f_{\varphi}^{(m)}\right) .
$$

Obviously, the mapping $\varphi \mapsto T_{\varphi}$ is a monomorphism of the $\operatorname{group} \varphi \in \operatorname{Aut}\left(\mathcal{L}(V), *_{A}\right)$ to the generalized wreath product $S=\operatorname{Aut}(\mathcal{L}(A)) \imath\left(S_{r_{0}}, S_{r_{1}}, \ldots, S_{r_{m}}\right)$. Now, we only need to show that this monomorphism is surjective.

Let $\tau=\left(\mu, f^{(0)}, f^{(1)}, \ldots, f^{(m)}\right)$ be an arbitrary subset from $S$. Then $\tau$ acts on the elements from $\mathcal{L}(V)$ by the next rule

$$
\tau(U)= \begin{cases}\mu(U), & \text { if } U \subseteq A ; \\ \mu(U \cap A) \dot{\cup} X_{f^{(\operatorname{dim}(U \cap A))}(U \cap A)(i)^{\prime}} & \text { if } U \nsubseteq A \text { and } U=(U \cap A) \dot{\cup} X_{i}^{(U)} .\end{cases}
$$

We represent an arbitrary element $W \in \mathcal{L}(A)$ as $W=W^{\prime} \dot{\cup} W^{\prime \prime}$, such as $W^{\prime}=W \cap A$ and $W^{\prime \prime}=W \backslash A$. Hence, for any $W_{1}, W_{2} \in \mathcal{L}(A)$ we have

$$
\tau\left(W_{1} *_{A} W_{2}\right)=\tau\left(\left(W_{1}^{\prime} \dot{\cup} W_{1}^{\prime \prime}\right) \cap A \cap\left(W_{2}^{\prime} \dot{\cup} W_{2}^{\prime \prime}\right)\right)=\tau\left(W_{1}^{\prime} \cap W_{2}^{\prime}\right)=\mu\left(W_{1}^{\prime} \cap W_{2}^{\prime}\right)=\mu\left(W_{1}^{\prime}\right) \cap \mu\left(W_{2}^{\prime}\right) .
$$


On the other hand, $\tau\left(W_{i}\right)=\mu\left(W_{i}^{\prime}\right) \dot{\cup} X_{i}$, where $X_{i} \cap A=\varnothing, i=1,2$. Thus,

$$
\tau\left(W_{1}\right) *_{A} \tau\left(W_{2}\right)=\left(\mu\left(W_{1}^{\prime}\right) \dot{\cup} X_{1}\right) \cap A \cap\left(\mu\left(W_{2}^{\prime}\right) \dot{\cup} X_{2}\right)=\mu\left(W_{1}^{\prime}\right) \cap \mu\left(W_{2}^{\prime}\right) .
$$

Hence, $\tau\left(W_{1} *_{A} W_{2}\right)=\tau\left(W_{1}\right) *_{A} \tau\left(W_{2}\right)$ and $\tau$ is the automorphism of the variant $\left(\mathcal{L}(V), *_{A}\right)$.

\section{References}

[1] Lyapin E.S. Semigroups. Fizmatgiz, Moscow, 1960. (in Russian)

[2] Chase K. Sandwich semigroups of binary relations. Discrete Math. 1979, 28 (3), 231-236. doi:10.1016/0012365X(79)90130-4

[3] Hickey J.B. Semigroups under a sandwich operation. Proc. Edinburg Math. Soc. 1983, 26 (3), 371-382. doi: $10.1017 /$ S0013091500004442

[4] Khan T.A., Lawson M.V. Variants of regular semigroups. Semigroup Forum 2001, 62 (3), 358-374. doi: $10.1007 / \mathrm{s} 002330010034$

[5] Mazorchuk V., Tsyaputa G. Isolated subsemigroups in the variants of $\mathcal{T}_{n}$. Acta Math. Univ. Comenian. (N.S.) 2008, $77(1), 63-84$.

[6] Ganyushkin O., Mazorchuk V. Classical Finite Transformation Semirgoups. An Introduction. In: Algebra and Applications, 9. Springer-Verlag, London, 2009. doi:10.1007/978-1-84800-281-4

[7] Desiateryk O. Variants of commutative bands with zero. Bull. T. Shevchenko National Univ. of Kyiv. Series: Phys. \& Math. 2015, 4, 15-20.

[8] Desiateryk O., Ganyushkin O. Variants of a lattice of partitions of a countable set. Algebra Discrete Math. 2018, 26 (1), 8-18.

Ганюшкін О.Г., Аесятерик О.О. Групи автоморфізмів деяких варіантів граток // Карпатські матем. публ. — 2021. — Т.13, №1. - С. 142-148.

У цій статті ми розглядаємо варіанти булеана і гратки підпросторів та досліджуємо їх групи автоморфізмів. Знайдено незвідні системи твірних варіантів гратки підмножин скінченної множини та гратки підпросторів скінченного векторного простору.

$\Delta$ оведено, що група автоморфізмів варіанта гратки підмножин скінченної множини $\epsilon$ вінцевим добутком двох симетричних груп підстановок, перша з яких діє на підмножинах, а група автоморфізмів варіанта гратки підпросторів скінченного векторного простору $є$ природним узагальненням вінцевого добутку, де перший множник $€$ групою автоморфізмів гратки підпросторів, а другий визначається певним набором симетричних груп.

Ключові слова і фрази: гратка, варіант, сендвіч-напівгрупа, група автоморфімів, булеан, гратка підпросторів, система твірних. 